\title{
Resource Partitioning by Mangrove Bird Communities in North Australia
}

\author{
Jayasilan Mohd-Azlan ${ }^{1,2}$, Richard A. Noske ${ }^{1}$, and Michael J. Lawes ${ }^{1,3}$ \\ ${ }^{1}$ Research Institute for the Environment and Livelihoods, Charles Darwin University, Darwin, Northern Territory, 0909 Australia \\ ${ }^{2}$ Department of Zoology, Faculty of Resource Science and Technology, Universiti Malaysia Sarawak, Kota Samarahan, Sarawak, 94300 \\ Malaysia
}

\begin{abstract}
Mangrove bird communities in north Australia comprise relatively few passerine species compared with other arboreal habitats in the region. Mangroves are dominated by a few tree species and there are potentially few resource axes available for partitioning by terrestrial birds. Competition for limited resources is predicted to cause strong niche differentiation and a highly structured, but low diversity, bird assemblage. Using multivariate and bipartite network analyses based on 1771 foraging observations (33\% of 5320 behavioral observations), we examined resource partitioning by 20 terrestrial bird species in mangroves of north Australia. The mangrove bird community largely comprised generalist insectivores that partitioned insects by size with moderate-to-high interspecific overlap in diet. Gleaning for insects was the most common foraging mode. Few species specialized on nectar. Flowers of one or more mangrove species were available in every month of the year and insect abundance was correlated with flowering peaks. Niche differentiation by birds was determined by food type and foraging mode more than by broad spatial (mangrove zones) or temporal (seasonal) segregation of the use of resources. There was little evidence of bird species saturation or species sorting, suggesting loose species packing and a lesser role than expected for species interactions and interference competition in structuring the bird assemblage in mangroves.
\end{abstract}

Key words: avifauna; bipartite network analysis; community ecology; niche segregation; plant-animal interactions.

Bird Communities have often BeEn used AS MODEL Systems to understand the complex interactions among species that use similar resources (Wooller \& Calver 1981, Ricklefs 2012). How species partition resources and differentiate their niches define how communities are structured (Schoener 1974, Greenberg \& Olsen 2010). Studies of how sympatric species differ in their resource use can provide indirect inferences about how potential competitors manage to coexist (Robertson et al. 2013). Coexisting species in an assemblage separate their niches or ecological needs by partitioning resources along temporal, spatial and behavioral niche axes (Cody 1974, Schoener 1974). In most instances where resources are limiting, competitive interactions among species lead to non-random patterns of resource use among species (Cody 1974). Some studies of closely related coexisting bird species, particularly in ecosystems with low vegetation diversity, have shown that bird species partition food resources by using different foraging behaviors (Lefebvre \& Poulin 1997, Kornan \& Adamik 2007, Böhm \& Kalko 2009, Greenberg \& Olsen 2010), vertical strata (Recher \& Davis 1998, Walther 2002, Styring \& Zakaria 2004) or by the differential use of microhabitats (Cody 1974, Schoener 1974). In the tropics, bird species are often more specialized in the way they use resources, which enables communities to be more tightly 'packed', and species richness is thus associated with specialization (MacArthur et al. 1972, Belmaker et al. 2012).

Received 9 October 2013; revision accepted 17 February 2014.

${ }^{3}$ Corresponding author; e-mail: Michael.Lawes@cdu.edu.au
However, one constraint on determining the role of species interactions on community assembly is obtaining sufficient information on all interactions in a species-rich assemblage, necessary to understand how resources are partitioned (Wheeler \& Calver 1996, Lewinsohn et al. 2006). Mangroves are a particularly suitable model ecosystem for examining species interactions because they are structurally and floristically simple, and support relatively few species of terrestrial vertebrates, so that niche differentiation may be more easily identified than in complex ecosystems, such as rain forests. Although the diets of many bird species of Australian mangroves have been described semi-quantitatively Johnstone 1990, Noske 2003), the relationship between food resource availability and niche partitioning has not been investigated ( $c f$. neotropical mangrove bird communities-Lefebvre \& Poulin 1997). The seasonal availability of food resources (i.e., insects and nectar) in mangroves is thought to influence bird species distribution and abundance in mangroves (Noske 1996) and its associated habitats (Woinarski 1993). In addition, the mangrove bird communities of northern Australia are particularly diverse (Ford 1982, Noske 1996) and have more mangrove-specialized species than those of other parts of the world (Luther \& Greenberg 2009, Nyári \& Joseph 2013), increasing the expectation of resource partitioning among Australian mangrove bird species (Noske 1996).

In Australia, mangroves represent a larger proportion of the land area of the monsoon tropics than monsoon rain forest. The monsoon tropics of northern Australia contain some of the most diverse mangrove communities in the world, yet there have been few studies of the phenology of mangrove flowering and insect abundance (Noske 1996, McGuinness 1997, Coupland et al. 